\title{
Cyclin-Dependent Kinase 5 Phosphorylates the N-Terminal Domain of the Postsynaptic Density Protein PSD-95 in Neurons
}

\author{
Maria A. Morabito, ${ }^{1}$ Morgan Sheng, ${ }^{2}$ and Li-Huei Tsai ${ }^{3}$ \\ ${ }^{1}$ Department of Pathology, Harvard Medical School, Boston, Massachusetts 02115, ${ }^{2}$ Picower Center for Learning and Memory, RIKEN-MIT Neuroscience \\ Research Center, Howard Hughes Medical Institute, Massachusetts Institute of Technology, Cambridge, Massachusetts 02139, and ${ }^{3}$ Department of \\ Pathology and Howard Hughes Medical Institute, Harvard Medical School, Boston, Massachusetts 02115
}

PSD-95 (postsynaptic density 95) is a postsynaptic scaffolding protein that links NMDA receptors to the cytoskeleton and signaling molecules. The N-terminal domain of PSD-95 is involved in the synaptic targeting and clustering of PSD-95 and in the clustering of NMDA receptors at synapses. The N-terminal domain of PSD-95 contains three consensus phosphorylation sites for cyclin-dependent kinase 5 (cdk5), a proline-directed serine-threonine kinase essential for brain development and implicated in synaptic plasticity, dopamine signaling, cocaine addiction, and neurodegenerative disorders.

We report that PSD-95 is phosphorylated in the N-terminal domain by cdk5 in vitro and in vivo, and that this phosphorylation is not detectable in brain lysates of cdk5-/ - mice. N-terminal phosphorylated PSD-95 is found in PSD fractions together with cdk5 and its activator, p35, suggesting a role for phosphorylated PSD-95 at synapses. In heterologous cells, coexpression of active cdk5 reduces the ability of PSD-95 to multimerize and to cluster neuronal ion channels, two functions attributed to the N-terminal domain of PSD-95. Consistent with these observations, the lack of cdk5 activity in cultured neurons results in larger clusters of PSD-95. In cdk5-/ - cortical neurons, more prominent PSD-95 immunostained clusters are observed than in wild-type neurons. In hippocampal neurons, the expression of DNcdk5 (inactive form of cdk5) or of the triple alanine mutant (T19A, S25A, S35A) full-length PSD-95 results in increased PSD-95 cluster size.

These results identify cdk5-dependent phosphorylation of the N-terminal domain of PSD-95 as a novel mechanism for regulating the clustering of PSD-95. Moreover, these observations support the possibility that cdk5-dependent phosphorylation of PSD-95 dynamically regulates the clustering of PSD-95/NMDA receptors at synapses, thus providing a possible mechanism for rapid changes in density and/or number of receptor at synapses.

Key words: phosphorylation; synapse; PSD-95; cdk5; NMDA receptor clustering; PSD-95 multimerization

\section{Introduction}

PSD-95/SAP-90 (synapse-associated protein-90) is a member of the membrane-associated guanylate kinase (MAGUK) family of proteins that has been implicated in the assembly of many components of the postsynaptic density (PSD) (Craven and Bredt, 1998; El-Husseini et al., 2000b; Garner et al., 2000; Kennedy, 2000; Lee and Sheng, 2000; Sheng and Pak, 2000; Craig and Boudin, 2001; McGee and Bredt, 2003). PSD-95 localizes and clusters at synapses early during synapse formation, suggesting that

\footnotetext{
Received 0ct. 9, 2003; revised Nov. 25, 2003; accepted Nov. 26, 2003.

This work was supported by National Institutes of Health Grants MH62161 (M.A.M.) and NS37007 (L.-H.T.) L.-H.T. is an Associate Investigator of the Howard Hughes Medical Institute. We thank Y. P. Hsueh for recombinant PSD-95 proteins, J. Ko for mutant mice brains, R. Neve for HSV recombinant viruses, Z. Xie for recombinant cdk5 kinase, L. Lanier and C. Sala for technical advice on hippocampal cultures, L. Edelmann for advice on confocal images, H. Hering and L. Moy for help with data analysis, and R. Dhavan, L. Orlando, and B. Samuels for reading this manuscript.

Correspondence should be addressed to Dr. Maria A. Morabito, University of Massachusetts Medical School Department of Cell Biology, E. K. Shriver Center, 200 Trapelo Road, Waltham, MA 02452. E-mail: Maria.Morabito@umassmed.edu.

DOI: 10.1523/JNEUROSCI.4582-03.2004

Copyright $\odot 2004$ Society for Neuroscience $\quad$ 0270-6474/04/240865-12\$15.00/0
}

PSD-95 plays a primary role in organizing and localizing proteins to the PSD (Rao et al., 1998), and thus contributes to synapse maturation (El-Husseini et al., 2000b). PSD-95 contains distinct structural domains such as three PDZ [PSD-95/Discs large (Dlg)/zona occludens-1 (ZO-1)] protein interaction motifs, an Src homology 3 (SH3) domain, and a C-terminal guanylate kinase domain. The PDZ domains of PSD-95 interact directly with intracellular signaling components (Brenman et al., 1996a; Chen et al., 1998; Kim et al., 1998), putative cell adhesion molecules (Irie et al., 1997), Shakertype $\mathrm{K}^{+}$channel (Kim et al., 1995), and NMDA receptors (NMDARs) (Kornau et al., 1995). Therefore, mechanisms that regulate synaptic clustering of PSD-95 may be fundamental to postsynaptic differentiation and efficacy of synaptic transmission. The $\mathrm{N}$ terminal domain of PSD-95 is essential for synaptic targeting and clustering of PSD-95 in neurons (Craven et al., 1999). The Nterminal domain of PSD-95 is palmitoylated (Milligan et al., 1995; Mumby, 1997; Topinka and Bredt, 1998), and this post-translational modification is required for its intracellular sorting, postsynaptic targeting, and ion channel clustering (Hsueh et al., 1997; Topinka and Bredt, 1998; Craven et al., 1999; El-Husseini et al., 2000a). 
We investigated the possibility that the phosphorylation of PSD-95 may also have an impact on its function. The N-terminal domain of PSD-95 contains three serine/threonine proline dipeptide motifs, which constitute minimal cyclin-dependent kinase 5 (cdk5) consensus phosphorylation sites (Beaudette et al., 1993; Songyang et al., 1996; Dhavan and Tsai, 2001). Cdk5 is a proline-directed serine/threonine kinase essential for brain development (Dhavan and Tsai, 2001). Cdk5 is active only when associated with one of its activators: p35 or its homolog p39. Cdk5-deficient mice and p35/p39 double knock-out mice die between late embryonic and perinatal stages (Ohshima et al., 1996; Ko et al., 2001). Cdk5, p35, and p39 are enriched in synaptic membranes and localize to postsynaptic compartments (Humbert et al., 2000; Niethammer et al., 2000). Recently, cdk5 has been shown to phosphorylate NR2A, a subunit of the NMDA receptor (NMDAR), which in turn influences long-term potentiation (LTP) induction in hippocampal neurons (Li et al., 2001). Using phospho-specific antibodies, we find that the N-terminal domain of PSD-95 is phosphorylated in vivo in a cdk5-dependent manner. We report that cortical neurons lacking cdk5 activity (cdk5-/- mutants) as well as hippocampal neurons expressing inactive cdk5 (DNcdk5) or the alanine mutant of PSD-95 have enlarged PSD-95 clusters. These studies indicate that phosphorylation by cdk 5 may negatively regulate the clustering of PSD-95 and NMDAR at synapses, thus implicating $\mathrm{cdk} 5$ in the regulation of synapse formation and synaptic plasticity.

\section{Materials and Methods}

DNA constructs. PSD-95 bacterial and mammalian expression constructs have been described previously (Hsueh et al., 1997). Mutagenesis of PSD-95 was performed by PCR amplification using Vent (New England Biolabs, Beverly, MA) and the appropriate oligonucleotides. All mutations were confirmed by sequencing. The mammalian constructs were expressed using the mammalian expression vectors GW1 or pcDNA3.

Kinase assays. The bacterial constructs were expressed in BL-21 and the recombinant His-tagged proteins purified using Ni-NTA agarose according to the manufacturer (Qiagen, Hilden, Germany). Bacterially produced histidine-tagged proteins were incubated with the p35-cdk5 kinase (immunoprecipitated from rat brain lysate) for $30 \mathrm{~min}$ at room temperature in the presence of $\left[\gamma^{32} \mathrm{P}\right.$ ATP $]$ as described previously (Nikolic et al., 1996). Alternatively, recombinant glutathione $S$-transferase (GST)-cdk5 and GST-p25 were incubated at room temperature for 20 min and then incubated in a 1:1:1 molar ratio (or with increasing amounts) of recombinant histidine-tagged proteins for increasing amounts of time (or for $5 \mathrm{~min}$ ) in the presence of $0.033 \times 10^{-3} \mathrm{mM}\left[\gamma^{32} \mathrm{P}\right.$ ATP] and $0.1 \mathrm{~mm}$ ATP. Ten percent of the samples were separated on 12 or $15 \%$ polyacrylamide gel, Coomassie stained, and autoradiographed or, alternatively, analyzed by PhosphorImager (Molecular Dynamics, Sunnyvale, CA) using ImageQuant.

Brain fractionations. All buffers contained a cocktail of protease and phosphatase inhibitors ( $1 \mathrm{~mm}$ PMSF, $1 \mu \mathrm{g} / \mathrm{ml}$ aprotinin, $10 \mu \mathrm{g} / \mathrm{ml} \mathrm{leu-}$ peptin, $1 \mu \mathrm{g} / \mathrm{ml}$ pepstatin, $1 \mathrm{~mm} \mathrm{Na}_{3} \mathrm{VO}_{4}, 50 \mathrm{~mm} \mathrm{NaF}$ ). Rat brain crude synaptosomal fraction (P2) and subcellular fractions were prepared as described previously (Huttner et al., 1983; Niethammer et al., 2000). Synaptosomal fractions were lysed in $150 \mathrm{~mm} \mathrm{NaCl}, 10 \mathrm{~mm}$ Tris, $\mathrm{pH}$ 8.0, and $1 \mathrm{~mm}$ EDTA with $1 \%$ SDS, followed by the addition of five volumes of 2\% Triton X-100, $150 \mathrm{~mm} \mathrm{NaCl}, 10 \mathrm{~mm}$ Tris, $\mathrm{pH}$ 8.0, and 1 mm EDTA and used for $\mathrm{p} 35$ coimmunoprecipitations, or lysed with $1 \%$ Triton X-100, $150 \mathrm{~mm} \mathrm{NaCl}, 10 \mathrm{~mm}$ Tris, pH 8, and $1 \mathrm{~mm}$ EDTA and used for Western blot analysis. Alternatively, synaptosomal fractions were lysed in radioimmunoprecipitation assay (RIPA) buffer and used for NR2B coimmunoprecipitation. Purified PSD fractions were prepared from rat brain lysate and subjected to detergent extraction as described previously (Cho et al., 1992). Synaptosomal fractions were extracted in $150 \mathrm{~mm}$ $\mathrm{NaCl}, 10$ mm Tris, $\mathrm{pH} 8.0$, and 1 mm EDTA in the presence of $0.5 \% \mathrm{NP}-40$ or $1 \%$ Triton X-100 and centrifuged at $100,000 \times g$. Pellets and supernatants were analyzed by SDS-PAGE and Western blot.

Phospho-epitope-specific antibodies. Phospho-epitope-specific antiserum against phosphorylated T19 and S25 PSD-95 was raised in rabbit using the synthetic phospho-peptide QDEDTPPLEHSPAH (Tufts Medical School, Boston, MA). The resulting antiserum was first purified from nonphosphorylated synthetic peptide coupled to a Sulfolink column (Pierce Biotechnology, Rockford, IL) and then affinity-purified over the phosphorylated T19 and S25 synthetic peptide linked to a Sulfolink column, according to the manufacturer's recommendation.

Immunoprecipitations and Western blotting. All buffers contained a cocktail of protease and phosphatase inhibitors ( $1 \mathrm{~mm}$ PMSF, $1 \mu \mathrm{g} / \mathrm{ml}$ aprotinin, $10 \mu \mathrm{g} / \mathrm{ml}$ leupeptin, $1 \mathrm{~mm} \mathrm{Na}_{3} \mathrm{VO}_{4}$, and $50 \mathrm{~mm} \mathrm{NaF}$ ). The brains of mice lacking p35, or both p35 and p39, or wild-type, were lysed in RIPA buffer. Adult rat brains were lysed in $150 \mathrm{~mm} \mathrm{NaCl}, 50 \mathrm{~mm}$ Tris, $\mathrm{pH} 7.5$, and $5 \mathrm{~mm}$ EDTA and immunoprecipitations were performed using anti-PSD-95 antibody (Upstate Biotechnology, Lake Placid, NY) or anti-Myc antibody (Oncogene, San Diego, CA) as control. COS-7 cells were lysed in RIPA buffer and immunoprecipitations were performed from transfected COS-7 cell lysates using anti-Myc antibody (Oncogene), or anti PSD-95-CSK, or anti-Kv1.4 antibodies. Complexes were isolated using protein A or protein G Sepharose. Tissue and cell lysates were separated by SDS-PAGE, transferred to Immobilon polyvinylidene difluoride membranes (Millipore, Billerica, MA) and immunoblotted with phospho-T19S25 antibody, anti-HA antibody (Santa Cruz Biotechnology, Santa Cruz, CA), anti-Myc antibody (Oncogene), anti-PSD-95 (Upstate Biotechnology), anti-Kv1.4, anti-p35 (Santa Cruz), and anticdk5 (Santa Cruz).

Cell cultures. COS-7 cells were maintained in DMEM supplemented with $10 \%$ fetal calf serum and transiently transfected with various plasmid constructs using Lipofectamine 2000 (Invitrogen, Grand Island, NY) according to the manufacturer's instructions. Cells were collected 16-20 hr after transfection.

Neuronal cultures. Pregnant animals were killed in accordance with institutional guidelines and as approved by the animal care and use committee. For cdk5-1- cultures, cortical neurons derived from embryonic day 16.5 (E16.5) brains were plated on coverslips coated with poly-Llysine at a density of $3.2 \times 10^{3} /$ coverslip and grown in neuronal conditioned Neurobasal medium (Invitrogen) supplemented with B27 (Invitrogen) and L-glutamine (Brewer et al., 1993). Neurons were fixed for immunostaining at $4 \mathrm{~d}$ in vitro (DIV).

Primary hippocampal cultures were prepared from E17-E18 rat brains and grown in Neurobasal medium supplemented with B27 and L-glutamine. Neurons were plated on poly-D-lysine and laminin at a density of 30,000/coverslip. Wild-type or phosphorylation mutant (T19A, S25A, S35A) full-length PSD-95 together with GFP were transfected after 14 DIV using Lipofectamine-2000 according to the manufacturer's instructions. Neurons were fixed and analyzed 16-20 hr after transfection. For the herpes simplex virus (HSV) infection experiments, neurons were plated on coverslips coated with poly-L-lysine at a density of 30,000/coverslip. Infections were performed using recombinant HSV expressing DNcdk5-GFP or LacZ (a gift from R. Neve, McLean Hospital, Belmont, MA) as described previously (Nikolic et al., 1996, 1998). Multiplicity of infection ranged from 0.1 to 1 . The efficiency of infection ranged from 28 to $70 \%$, depending on the HSV preparation. Neurons were fixed for immunostaining $18-20 \mathrm{hr}$ after infection.

Immunostaining. COS-7 cells were fixed in $4 \%$ paraformaldehyde for $20 \mathrm{~min}$ at room temperature followed by permeabilization with $0.1 \%$ Triton X-100 and 10\% BSA in PBS. Hippocampal and cortical neurons were fixed in $-20^{\circ} \mathrm{C}$ methanol for $10 \mathrm{~min}$, followed by permeabilization with $0.2 \%<$ Triton X-100 and 10\% BSA in PBS. Hippocampal neurons used for p35 and PSD-95 immunostaining were fixed in 4\% paraformaldehyde for $20 \mathrm{~min}$ at room temperature. Immunostaining was performed using anti-PSD-95 (guinea pig anti-PSD-95; M. Sheng), antiKv1.4 (M. Sheng), anti-NR1 (PharMingen International, San Diego, CA), and anti-p35 (Santa Cruz). Coverslips were mounted in ProLong antifade (Molecular Probes, Eugene, OR) and analyzed using Nikon (Tokyo, Japan) Delta Vision microscopy. Cortical cultures were analyzed blind by Carl Zeiss (Oberkochen, Germany) Axiovert 100M confocal 

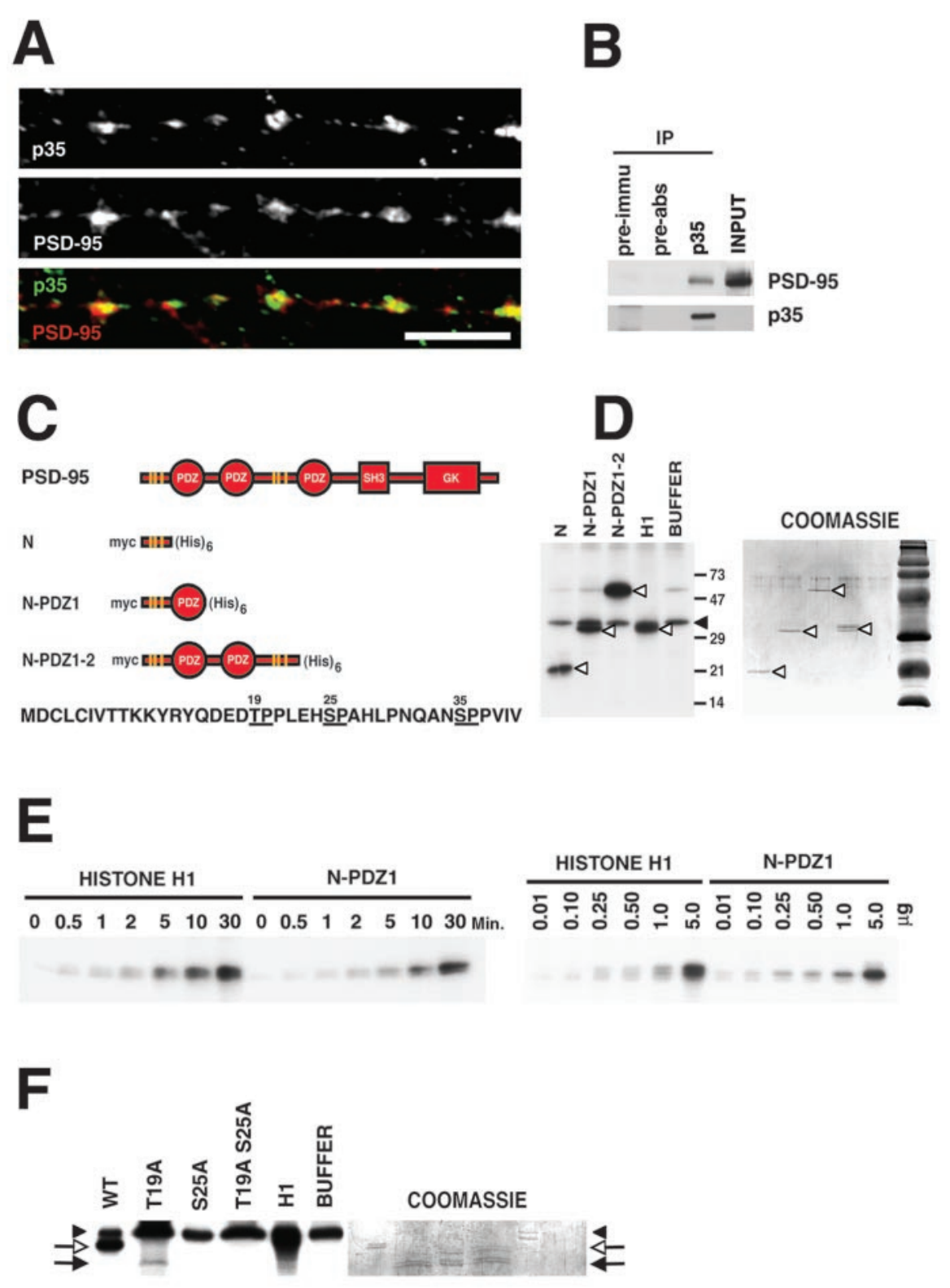

Figure 1. PSD-95 associates with $\mathrm{p} 35$ and is an in vitro substrate of p35-cdk5. A, Colocalization of p35 and PSD-95 along dendrites of hippocampal neurons. Hippocampal neurons (14 DIV) were fixed and immunostained for p35 and PSD-95. Scale bar, $10 \mu \mathrm{m}$. B, Coimmunoprecipitation of $\mathrm{p} 35$ and PSD-95 from synaptosome lysates. Synaptosomes were lysed in the presence of $1 \%$ SDS, and immunoprecipitations were performed using polyclonal $\mathrm{p} 35$ antiserum, antiserum preabsorbed with p35 antigen, or preimmune serum. C, Constructs used and the amino acid sequence of the $\mathrm{N}$-terminal domain of PSD-95. All constructs contain the $\mathrm{N}$-terminal domain with the three $\mathrm{cdk} 5$ consensus phosphorylation sites (T19, S25, S35). D, In vitro kinase assay using p35-cdk5 immunoprecipitated from brain. N, N-PDZ1, and N-PDZ1-2S were bacterially expressed as Myc-His-tagged proteins and $1 \mu \mathrm{g}$ of each protein was used as a substrate in an in vitro kinase assay using p35-cdk5 immunoprecipitated from brain. Histone H1 (H1), a generic substrate of $\mathrm{p} 35$-cdk5, was used as positive control. The white arrowheads indicate the recombinant proteins; the black arrowhead indicates autophosphorylated $\mathrm{p} 35$. The Coomassie staining indicates the amount of proteins used. $E$, In vitro kinase assay using purified reconstituted recombinant cdk5 kinase. Left, GST-p25, GST-cdk5, and substrate (either N-PDZ1 or histone H1 as control) were added to the reaction to a final concentration of $6.6 \times 10^{-7} \mathrm{M}$. The kinetics of the reactions were determined by analyzing the incorporation of ${ }^{32} \mathrm{P}$ ATP after increasing incubation periods. Right, Increasing amounts of N-PDZ1 or histone H1 were used as substrates in the in vitro kinase reaction with reconstituted recombinant cdk5. The reaction was stopped after $5 \mathrm{~min}$ by adding sample buffer. F, In vitro kinase assay of PSD-95 mutants. N-PDZ1 mutants (T19A, S25A, and T19A S25A) were expressed as His-tagged proteins in bacteria and $1 \mu \mathrm{g}$ of each was used in an in vitro kinase assay using $\mathrm{p} 35$-cdk5 kinase immunoprecipitated from brain. The mutant proteins are not Myc-tagged, and they display a faster mobility on SDS-PAGE. The wild-type recombinant protein and the mutant proteins are indicated by the white and black arrows, respectively, and the arrowhead indicates autophosphorylated 35. microscopy using a $25 \times$ objective. TOTO- 3 Iodide (Molecular Probes) was used to stain nuclei. PSD-95 immunostaining were quantified blind using Metamorph (Downingtown, PA) software using a threshold of 90 . A total of 32 hippocampal neurons transfected with wildtype full-length PSD-95 and 32 hippocampal neurons transfected with mutant (T19A, S25A, S35A) full-length PSD-95 were analyzed blind by Zeiss confocal microscopy. PSD-95 immunostaining was quantified blind using Metamorph software at a threshold of 120. Infected hippocampal neurons were analyzed using Nikon Delta Vision microscopy. A total of 431 (infected with HSVDNcdk5-GFP) and 371 (infected with HSVLacZ) PSD-95 immunoreactive regions derived from 14 and 8 dendrites, respectively, were analyzed blind by Metamorph software using a threshold of 94. The areas of immunostaining were rendered in number of pixels. The statistical significance of this analysis was determined using the unpaired $t$ test.

\section{Results}

PSD-95 is a substrate of the

\section{p35-cdk5 kinase}

The N-terminal region of PSD-95 contains three $\mathrm{cdk} 5$ consensus phosphorylation sites, suggesting the possibility that PSD-95 is a substrate of cdk5. Three additional potential phosphorylation sites are within the linker region between the second and third PDZ motif. p35 and cdk5 are present in the PSD (Niethammer et al., 2000), and p35 colocalizes with PSD-95 along the dendrites of primary hippocampal neurons (Fig. $1 A$ ). An interaction between p35-cdk5 and PSD-95 is supported by the association of p35 and PSD-95 in synaptosomes, as detected by coimmunoprecipitation of PSD-95 with a p35 antibody (Fig. $1 B$ ).

To investigate whether the interaction between p35 and PSD-95 results in phosphorylation of PSD-95 by cdk5, 6X histidine-tagged PSD-95 recombinant proteins were used as substrates for in vitro kinase assays using either p35-cdk5 immunoprecipitated from brain (Fig. $1 D$ ), or kinase reconstituted from recombinant GST-cdk5 and GST-p25 (Fig. 1E). Deletion mutants Myc-N-PDZ1-2S-His, Myc$\mathrm{N}-\mathrm{PDZ} 1-\mathrm{His}$, and Myc-N-His contain only the N-terminal three potential cdk5 phosphorylation sites, T19, S25, and S35 (Fig. 1C,D) (Hsueh et al., 1997). These fusion proteins were readily phosphorylated by immunoprecipitated $\mathrm{p} 35$-cdk5 in vitro to a extent similar to that of histone $\mathrm{H} 1$, a generic substrate of cdk5 (Fig. 1D). To exclude the possibility that other coimmunoprecipitated kinases may contribute to the phosphorylation, an in vitro kinase assay was performed using cdk5 reconstituted from recombinant GST-cdk5 and GST-p25. Myc-N-PDZ1-His or his- 
tone $\mathrm{H} 1$ were used as substrates and the reactions were performed with a $1: 1: 1$ stoichiometry of GST-p25, GST-cdk5, and Myc-N-PDZ1-His or histone $\mathrm{H} 1$ (Fig. $1 E$, left). The phosphorylation of Myc-N-PDZ1-His, similar to histone $\mathrm{H} 1$, can be detected as early as $2-5 \mathrm{~min}$ after the start of the reactions. However, when increasing amounts of substrate were used and the kinase assays were performed for only $5 \mathrm{~min},{ }^{32} \mathrm{P} \gamma \mathrm{ATP}$ incorporation increased proportionally to the increase in recombinant $\mathrm{Myc}-\mathrm{N}$ PDZ1-His protein added to the reaction, further supporting the specificity of the phosphorylation (Fig. $1 E$, right). To further confirm PSD-95 as an in vitro substrate of $\mathrm{cdk} 5$, the stoichiometry of the reaction was calculated by using recombinant p25-cdk5. ATP (58.468 pmol) was incorporated into recombinant Myc-N-PDZ1-2S-His (67.7 pmol) after $1 \mathrm{hr}$, to a stoichiometry of 0.863 , comparable with the stoichiometry of the phosphorylation of protein phosphatase inhibitor-1 by cdk5 (Bibb et al., 2001). Together, these experiments indicate that the N-terminal domain of PSD-95 is a specific substrate of cdk5 in vitro. Among the three potential phosphorylation sites in the $\mathrm{N}$-terminal region of PSD-95, S25 (SPAH) is an optimal cdk5 phosphorylation site (Fig. $1 C$ ). To investigate which of the three sites (T19, S25, and S35) is phosphorylated by cdk5, an in vitro p35-cdk5 kinase assay was performed using alanine substitution mutants of N-PDZ1-His proteins and p35cdk5 immunoprecipitated from brain. In this assay, the single mutant T19A was phosphorylated by cdk5, although to a much lesser extent than the wild-type protein, whereas the S25A and the T19A S25A mutants were not phosphorylated to detectable levels (Fig. $1 F$ ). The observed difference in migration between mutant and wild-type proteins is caused by the absence of the Myc tag in the mutants. These results indicate that S25 and T19 are in vitro phosphorylation sites and S25 is the primary target of phosphorylation by cdk5.

To evaluate whether T19 and S25 are phosphorylated in vivo, a phospho-epitope-specific antibody (phospho-T19S25) was raised against a synthetic peptide containing both phospho-T19 and phospho-S25 (Fig. 2A). Phospho-T19S25 antibody recognized endogenous PSD-95 immunoprecipitated from rat brain lysates by a monoclonal PSD-95 antibody (Fig. 2B). The phospho-T19S25 antibody recognizes the band with the second slower mobility (Fig. 2C). The specificity of the purified phospho-T19S25 antibody was verified by treatment with calf intestine phosphatase (CIP) of immunoprecipitated PSD-95. This treatment reduced the ability of the phospho-T19S25 antibody to recognize PSD-95 (Fig. 2C). In addition, the specificity of the antibody was tested by Western blotting of COS-7 cell lysates
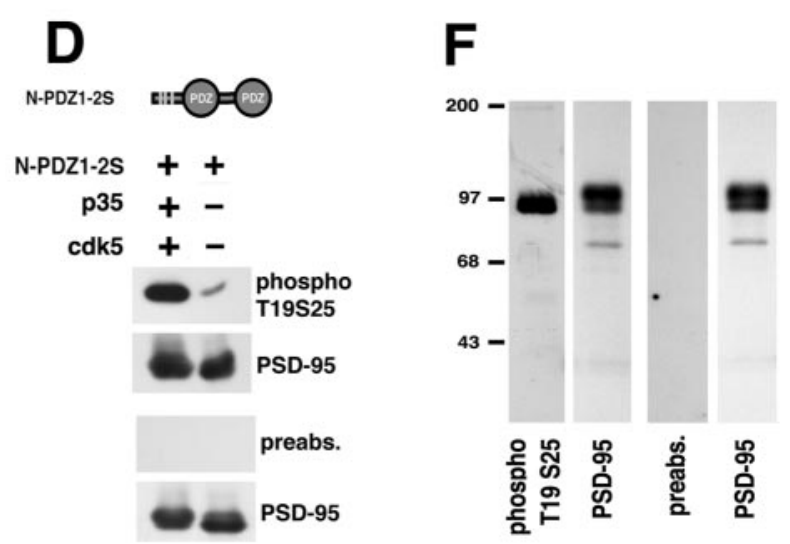

Figure 2. $\quad$ PSD-95 is phosphorylated in vivo in a p35-cdk5-dependent manner. $A$, Sequence of the peptide used to raise the phospho-epitope-specific antibody. T19 and S25 are both phospho-residues. B, Western blot of endogenous PSD-95 using the 作 25 antibody. Endogenous PSD-95 was immunoprecipitated with PSD-95 antibody from rat brain lysates and blot作 antibody. Antibody preabsorbed with the phospho-peptide (phosphorylated T19 and S25) was used as a control. The blot S25A) in the presence (or absence) of $\mathrm{p} 35$-cdk5. The blot was probed with phospho-T19S25 antibody and subsequently probed with PSD-95, p35, and cdk5 antibodies. F, Western blot of rat synaptosomal lysates. The lysates were immunoblotted for phosphoT19S25 antibody, or phospho-T19S25 antibody preabsorbed with the phospho-peptide. PSD-95 antibody was used as control for loading. G, Western blot of E17 mutant and wild-type mouse brain lysates. The phosphorylation on T19 and S25 of endogenous PSD-95 was evaluated in brain lysates of wild-type, p35-/ - p39+/+, or p35-/ - p39-/ - mice. Lysates were immunoblotted with phospho-T19S25 antibody and subsequently probed for PSD-95 as a loading control.

coexpressing truncated PSD-95 (N-PDZ1-2S) and p35-cdk5. Although phospho-T19S25 antibody hardly detected PSD-95 when expressed alone, it generated a robust signal in lysates from cells expressing PSD-95 and p35-cdk5 (Fig. 2D). Preabsorption of this antibody with the antigenic peptide completely abolished the ability of the phospho-T19S25 antibody to recognize PSD-95 (Fig. 2D). To further determine the specificity of the phosphoT19S25 antibody, T19A and S25A N-PDZ1-2S mutants were expressed in COS-7 cells in the absence or presence of p35-cdk5. Western blot analysis of lysates reveals that although the phospho-T19S25 antibody detected a strong PSD-95 signal in lysates expressing wild-type protein, the signal was abolished in lysates expressing the S25A mutant and was markedly reduced in lysates expressing the T19A mutant (Fig. 2E). Together, these data indicate that the phospho-T19S25 antibody recognizes both phospho-T19 and phospho-S25, with phosphoS25 likely to be the major recognition epitope. 
In synaptosomal fractions from rat brain lysates, the phospho-T19S25 antibody detected a single band of the expected molecular weight for PSD-95, whereas no immunoreactive band was observed when the antibody was preabsorbed with the phospho-peptide used as antigen (Fig. 2). A slower migrating form of PSD-95 not recognized by the phospho-T19S25 antibody was also observed (Fig. $2 \mathrm{~F}$ ), possibly representing a product of other post-translational modifications or the alternatively spliced isoform of PSD-95 (Chetkovich et al., 2002). These results suggest that PSD-95 is phosphorylated on T19, S25, or both, in vivo. In addition, they indicate that the phospho-T19S25 antibody is highly specific to phosphorylated PSD-95 in synaptosomes. To verify that the phosphorylation of PSD-95 was dependent on cdk5 activity, the contribution of cdk 5 to the phosphorylation of endogenous PSD-95 was evaluated in brain lysates derived from E17 p35-/- and p35-/- p39-/- mice. Reduced phosphoT19-S25 immunoreactivity was observed in p35-/- lysates, and no strong signal was detected in lysates of p35-/- p39-/ - mice, which lack cdk5 activity (Fig. $2 G$ ), although comparable levels of PSD-95 were present in mutant and wild-type brains (Fig. 2G). These observations strongly suggest that PSD-95 is a physiological substrate of cdk5.

\section{Phosphorylated PSD-95 is present in the PSD}

PSD-95 is a protein targeted to synapses and enriched at the PSD. To assess the subcellular distribution of phosphorylated PSD-95, biochemical fractionation of adult brain lysates was performed (Fig. 3A). PSD-95 was abundant in the crude synaptosomal fraction (P2) and in the synaptosomal membrane fraction (LP1). Overall, the subcellular distribution of phospho-PSD-95 was reminiscent of that of total PSD-95. Because PSD-95 is abundant in PSD fractions (Cho et al., 1992), these fractions were analyzed for the presence of phosphorylated PSD-95. The phosphorylated form of PSD-95 was enriched in PSD fraction II and in the core of PSD (fraction III) (Fig. 3B). This distribution pattern resembled that of p35 and cdk5 (Fig. 3B), consistent with the possibility that phosphorylation of PSD-95 occurs postsynaptically and that it plays a role in synaptic function. PSD-95 is an itinerant vesicular protein, and its association with membranes mediates its intracellular trafficking (El-Husseini et al., 2000a). To investigate the possibility that phosphorylation of the $\mathrm{N}$-terminal domain of PSD-95 may alter its association with membranes, synaptosomal membranes were extracted with non-ionic detergents and the derived fractions analyzed by western blot (Fig. 3C). Phosphorylated PSD-95 was more readily extracted than the total PSD-95; $25.27 \%$ of total PSD-95 was soluble in $0.5 \%$ NP-40 and $13.74 \%$ in $1 \%$ Triton $\mathrm{X}-100$. In contrast, as detected by the phosphoT19S25 antibody, $68.50 \%$ of phosphorylated PSD-95 was soluble in $0.5 \%$ NP-40 and $45.46 \%$ in $1 \%$ Triton X-100. The increased extractability of phosphorylated PSD-95 versus total PSD-95 suggests that the phosphorylation of the $\mathrm{N}$ terminal of PSD-95 results in a weaker attachment of PSD-95 to membranes compared with the unphosphorylated form.

\section{Phosphorylation regulates PSD-95 multimerization but not neuronal ion channel binding} PSD-95 functions as a scaffolding protein that organizes large protein complexes at synapses. The N-terminal domain of PSD-95 mediates its multimerization (Hsueh et al., 1997), whereas the first two PDZ domains bind specifically to the $\mathrm{C}$ terminal of Shaker-type $\mathrm{K}^{+}$channels (Kv1.4) and of NR2 subunits of NMDARs (Kim et al., 1995; Kornau et al., 1995; Niethammer et al., 1996). To investigate whether phosphoryla-
A
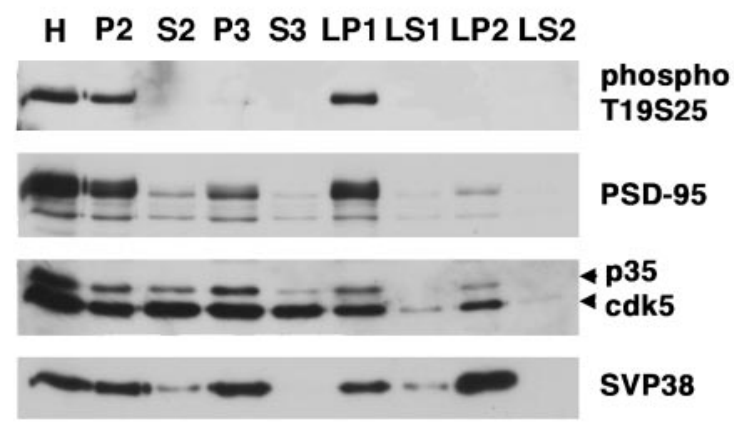

B

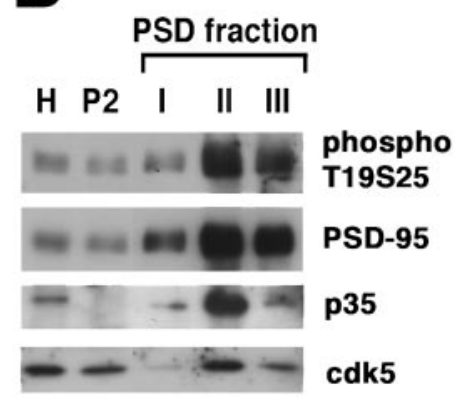

C

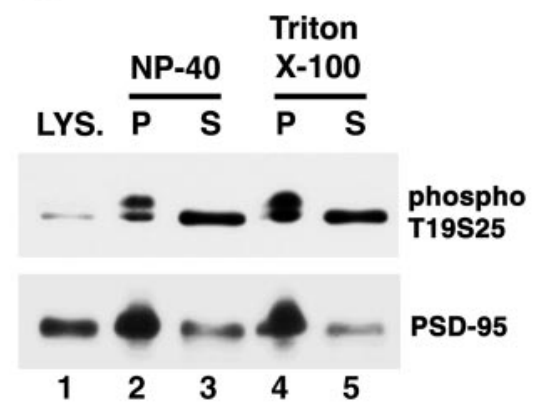

Figure 3. Phosphorylated PSD-95 is present in synaptosomal membrane fraction LP1 and in PSD fractions. A, Western blot of subcellular fractions of rat brain lysates. The blot was probed with phospho-T19S25 antibody and, subsequently, with antibodies for PSD-95, synaptophysin (SVP38), p35, and cdk5. H, Brain lysate; P2, crude synaptosomal fraction; S2, cytosol and light membranes; P3m light membranes (Golgi and ER); S3, cytosolic fraction; LP1, synaptosomal membrane fraction; LS1, synaptosomal cytosol; LP2, vesicles and small membrane fractions; LS2, supernatant of LP2. B, Western blot of PSD fractions. PSD fractions were prepared from P2 by differential and density gradient centrifugation followed by extraction once with Triton X-100 (I), twice with Triton X-100 (II), or once with Triton X-100 and once with N-lauroylsarcosine (III). Fraction III represents the core of PSD. One microgram of protein was loaded for each fraction of the PSD. The blot was probed with phospho-T19S25 antibody and, subsequently, with antibodies for PSD-95, p35, and cdk5. C, Detergent extractions of synaptosomal fractions. Crude synaptosomal fractions were extracted with either $0.5 \%$ NP-40 or $1 \%$ Triton $\mathrm{X}$-100 followed by $100,000 \times g$ centrifugation. The soluble and pellet fractions were blotted with phospho-T19S25 antibody and, subsequently with PSD-95 antibody.

tion of the N-terminal domain of PSD-95 affects association of PSD-95 with ion channels, coimmunoprecipitations were performed from lysates of COS-7 cells coexpressing N-PDZ1-2S and $\mathrm{Kv} 1.4$, in the presence or absence of $\mathrm{p} 35$-cdk5. In these lysates, phosphorylation of PSD-95 by p35-cdk5 was evident from the 
presence of the slower-migrating species of PSD-95. An antibody against Kv1.4 coimmunoprecipitated PSD-95, regardless of p35-cdk5 expression (Fig. $4 A$, top). This result suggests that phosphorylation of the N-terminal domain of PSD-95 does not disrupt the association of PSD-95 with Kv1.4. In addition, in synaptosomal lysates, phosphorylated PSD-95 is coimmunoprecipitated by an antibody against NR2B, a subunit of the NMDAR that binds specifically to the first two PDZ domains of PSD-95, as detected by phosphoT19S25 antibody (Fig. 4A, bottom). Together, these observations support the possibility that PSD-95 binds neuronal ion channels irrespective of the phosphorylation of the N-terminal domain of PSD-95. Because the N-terminal domain of PSD-95 has been implicated in the multimerization of PSD-95, it is possible that the phosphorylation of this region of PSD-95 by cdk5 may affect the self-association of PSD-95. This possibility was tested in COS-7 cells using PSD-95 constructs that are either of different size or have a different epitope tag. In one set of experiments, COS-7 cells were cotransfected with Myctagged full-length PSD-95 (Myc-PSD-95), untagged N-PDZ1-2S, and with or without p35-cdk5. As predicted, the anti-Myc antibody, which recognizes only the fulllength PSD-95, coimmunoprecipitated N-PDZ1-2S in the absence of p35-cdk5. However, when p35 and cdk5 were coexpressed, coimmunoprecipitation of N-PDZ1-2S with Myc-PSD-95 by the anti-Myc antibody was drastically reduced (Fig. $4 \mathrm{~B}$ ), suggesting that phosphorylation of N-PDZ1-2S and MycPSD-95 by p35-cdk5 decreases PSD-95 multimerization. This observation was confirmed using two differentially tagged full-length PSD-95 constructs, either HA- or Myc-tagged (HA-PSD-95 and Myc-PSD95, respectively). The anti-Myc antibody coimmunoprecipitated HA-PSD-95 with Myc-PSD-95 in the absence of p35-cdk5 (Fig. 4C). However, when p35 and cdk5 were coexpressed, coimmunoprecipitation of HA-PSD-95 with Myc-PSD-95 was markedly reduced (Fig. $4 C$ ). To verify that the phosphorylation of the N-terminal domain of PSD-95 by p35-cdk5 was responsible for the observed reduction of PSD-95 multimerization, the triple alanine phosphorylation mutants (T19A, S25A, S35A) of full-length PSD-95 (AAA PSD-95) and N-PDZ1-2S (AAA N-PDZ1-2S) were cotransfected with or without p35-cdk5 in COS-7 cells (Fig. 4D). AAA PSD-95 was immunoprecipitated by an antibody raised against the $\mathrm{SH} 3$ domain of PSD-95
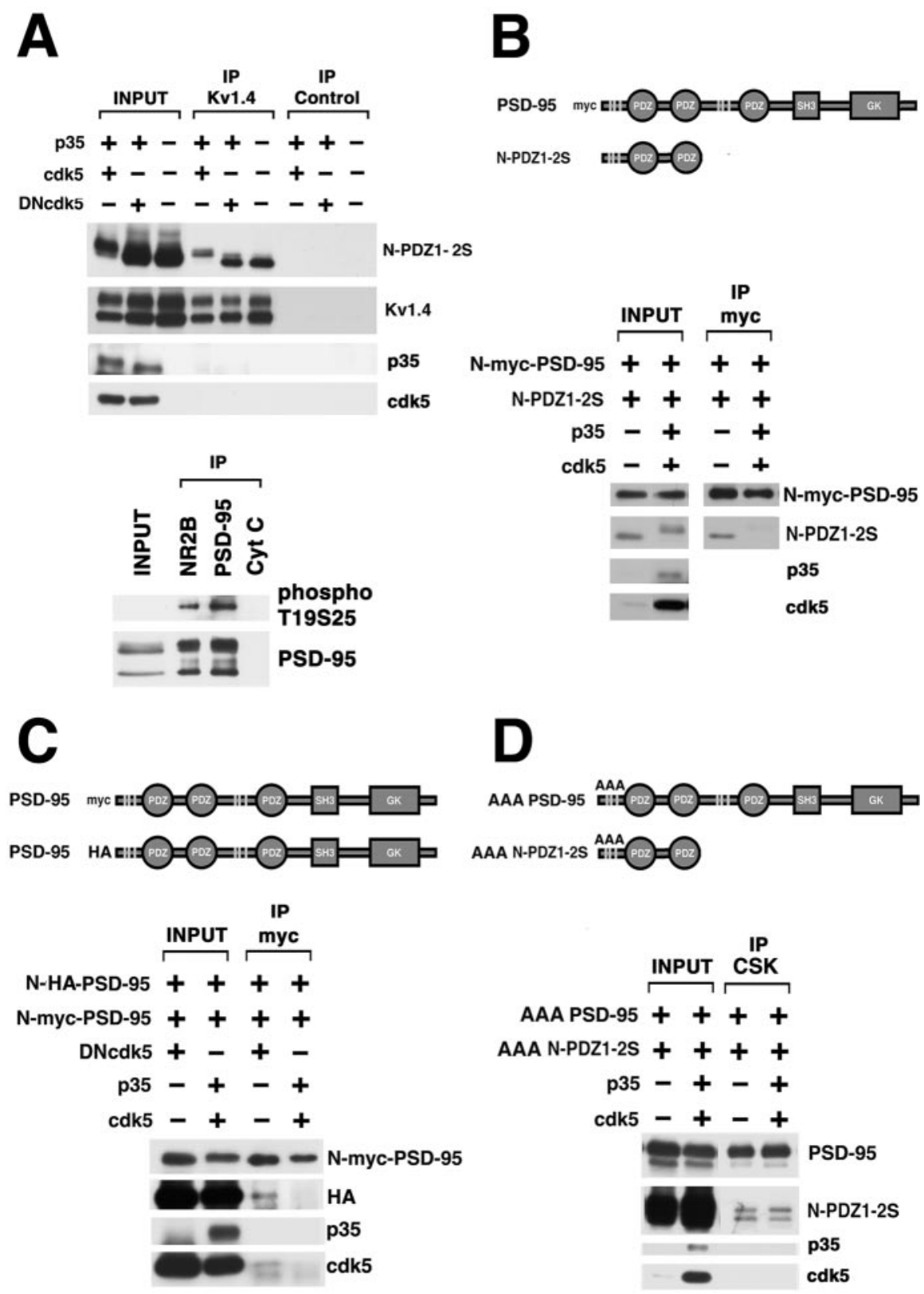

Figure 4. Phosphorylation of the N-terminal domain of PSD-95 by cdk5 reduces PSD-95 multimerization but not ion channel binding. A, top, Coimmunoprecipitation of phosphorylated PSD-95 with Kv1.4 channel. Kv1.4 antibody was used to coimmunoprecipitate PSD-95 from lysates of COS-7 cells cotransfected with PSD-95 (N-PDZ1-2S) and Kv1.4, in the presence (or absence) of p35-cdk5, or with inactive cdk5 (DNcdk5). Monoclonal PSD-95 antibody was used to detect PSD-95. The slow PSD-95 mobility band is diagnostic of phosphorylation. Kv1.4, cdk5, and p35 antibodies were used as controls. Bottom, Coimmunoprecipitation of phosphorylated PSD-95 with NMDA receptor. NR2B antibody was used to coimmunoprecipitate PSD-95 from synaptosomal lysates. PSD-95 and cytochrome ( (Cyt C) antibodies were used as controls. Phospho-T19S25 and monoclonal PSD-95 antibodies were used to detect phosphorylated and total PSD-95, respectively. $B$, Effect of phosphorylation by cdk5 on coimmunoprecipitation of PSD-95. Myc-PSD-95 was immunoprecipitated with anti-Myc antibody from lysates of COS-7 cells expressing N-PDZ1-2S and Myc-tagged full-length PSD-95 with (or without) p35-cdk5. The immunoprecipitates were blotted with anti-PSD-95 antibody. N-PDZ1-2S and Myc-tagged full-length PSD-95 proteins were identified by their differential mobility on SDS-PAGE. The blot was subsequently probed with p35 and cdk5 antibodies. Input lanes were loaded with $10 \%$ of lysates. C, Effect of phosphorylation by cdk5 on coimmunoprecipitation of HA- and Myc-tagged PSD-95. Myc-PSD-95 was immunoprecipitated with anti-Myc antibody from lysates of COS-7 cells expressing HA- and Myc-tagged full-length PSD-95 with (or without) p35-cdk5. The immunoprecipitates were blotted with anti-HA antibody, and subsequently probed with Myc, p35, and cdk5 antibodies. Input lanes were loaded with $10 \%$ of lysates. D, Coimmunoprecipitation of the alanine mutants of PSD-95. Full-length PSD-95 was immunoprecipitated with the CSK antibody from lysates of COS-7 cells expressing the triple alanine mutant (T19A, S25A, S35A) of N-PDZ1-2S and full-length PSD-95. CSK antibody was raised against the SH3 domain of PSD-95 and does not recognize N-PDZ12S. The immunoprecipitates were blotted with anti-PSD-95 antibody. N-PDZ1-2S and full-length PSD-95 were identified by their differential mobility on SDS-PAGE. The blot was subsequently probed with $\mathrm{p} 35$, and cdk5 antibodies. Input lanes were loaded with $10 \%$ of lysates. 
(Hsueh and Sheng, 1999). AAA N-PDZ1-2S coimmunoprecipitated with AAA PSD-95 regardless of p35-cdk5 expression (Fig. $4 D$ ). Together, these experiments provide evidence that phosphorylation of the N-terminal domain of PSD-95 by p35-cdk5 reduces $\mathrm{PSD}-95$ multimerization.

\section{Phosphorylation of PSD-95 regulates clustering of PSD-95 and ion channels in COS-7 cells}

PSD-95 is a scaffolding protein that anchors NMDAR at synapses. In heterologous cells, the coexpression of PSD-95 with ion channels such as Kv1.4 and NMDAR induces their coclustering (Kim et al., 1995; Kim and Sheng, 1996). The truncated form of PSD-95, N-PDZ1-2S, has been shown to cluster Kv1.4 as effectively as full-length PSD-95 (Hsueh et al., 1997). To explore the possibility that phosphorylation of PSD- 95 by p 35 -cdk 5 regulates the clustering of PSD-95 and ion channels, COS-7 cells were cotransfected with N-PDZ1-2S, Kv1.4, and either GFP alone, p35-cdk5-GFP, or inactive kinase (DNcdk5-GFP) (Nikolic et al., 1996, 1998) (Fig. 5A). As predicted, $72 \%$ of COS-7 cells coexpressing Kv1.4 and N-PDZ1-2S displayed coclustering of PSD-95 and Kv1.4 (Fig. 5A, $a-d$ ). However, only 3.2\% of cells cotransfected with p35-cdk5-GFP displayed immunostained clusters of PSD-95 and Kv1.4 (Fig. 5A, $e-h$ ). Consistent with these results, $68 \%$ of transfected cells expressing DNcdk5-GFP showed clusters of PSD-95 and Kv1.4 (Fig. 5A, $i-l$ ). The scoring and quantification of these experiments was performed in a blind manner. The images were taken focusing on the cell surface and the PSD-95 clusters colocalize with the Kv1.4 clusters, suggesting a surface expression of these clusters, although we cannot exclude the possibility that some of these clusters may not be at the cell surface. Together, these results suggest that phosphorylation of PSD-95 by cdk 5 reduces coclustering of PSD-95 and ion channels in heterologous cells. To further address whether N-terminal phosphorylation of PSD-95 is responsible for the observed reduction of clustering, the triple alanine mutant of truncated PSD-95 (T19A, S25A, S35 NPZ1-2S) and Kv1.4 were expressed in COS-7 cells with, or without, p35-cdk5-GFP (Fig. 5B). Like the wild-type protein, the triple alanine mutant of PSD-95 formed clusters with Kv1.4 (Fig. 5B, $a-c$ ). However, unlike the wild-type PSD-95 protein, expression of p35-cdk5 did not affect the clustering of mutant PSD-95 and Kv1.4 (Fig. 5B, $d-f$ ). These data argue for a role of phosphorylation of the N-terminal domain of PSD-95 by p35$\mathrm{cdk} 5$ in the regulation of coclustering of PSD-95 and neuronal ion channels.

\section{Cdk5-/- embryonic cortical neurons display enhanced PSD-95 clusters}

To evaluate clustering of PSD-95 in mice lacking cdk5 activity, the PSD-95 staining pattern was analyzed in cultured cortical neurons derived from $\mathrm{cdk} 5-/-$ and wild-type littermates. Cdk5-/- mice are perinatal lethal, and the neurons derived from them do not thrive in low-density long-term cultures. To analyze the PSD-95 clusters in these mice, cortical neurons were cultured for 4 DIV. Although synaptogenesis is still active in these young cultures and most of the synapses are not yet fully formed, larger PSD-95 immunostained clusters were observed in cdk5-/- neurons (Fig. 6A, $b, d$ ) compared with wild-type cultures (Fig. $6 A, a, c$ ). The areas of PSD-95 immunostaining were quantified blind and rendered in number of pixels. In wild-type neurons, small PSD-95 clusters (10-30 pixels) were $75.82 \%$ of total clusters (SEM, 5.50\%) versus the $43.53 \%$ (SEM, 10.98\%) observed in cdk5-/- neurons (Fig. 6 B). Conversely, larger clusters were more frequent in $\mathrm{cdk} 5-/-$ than in wild-type neurons.
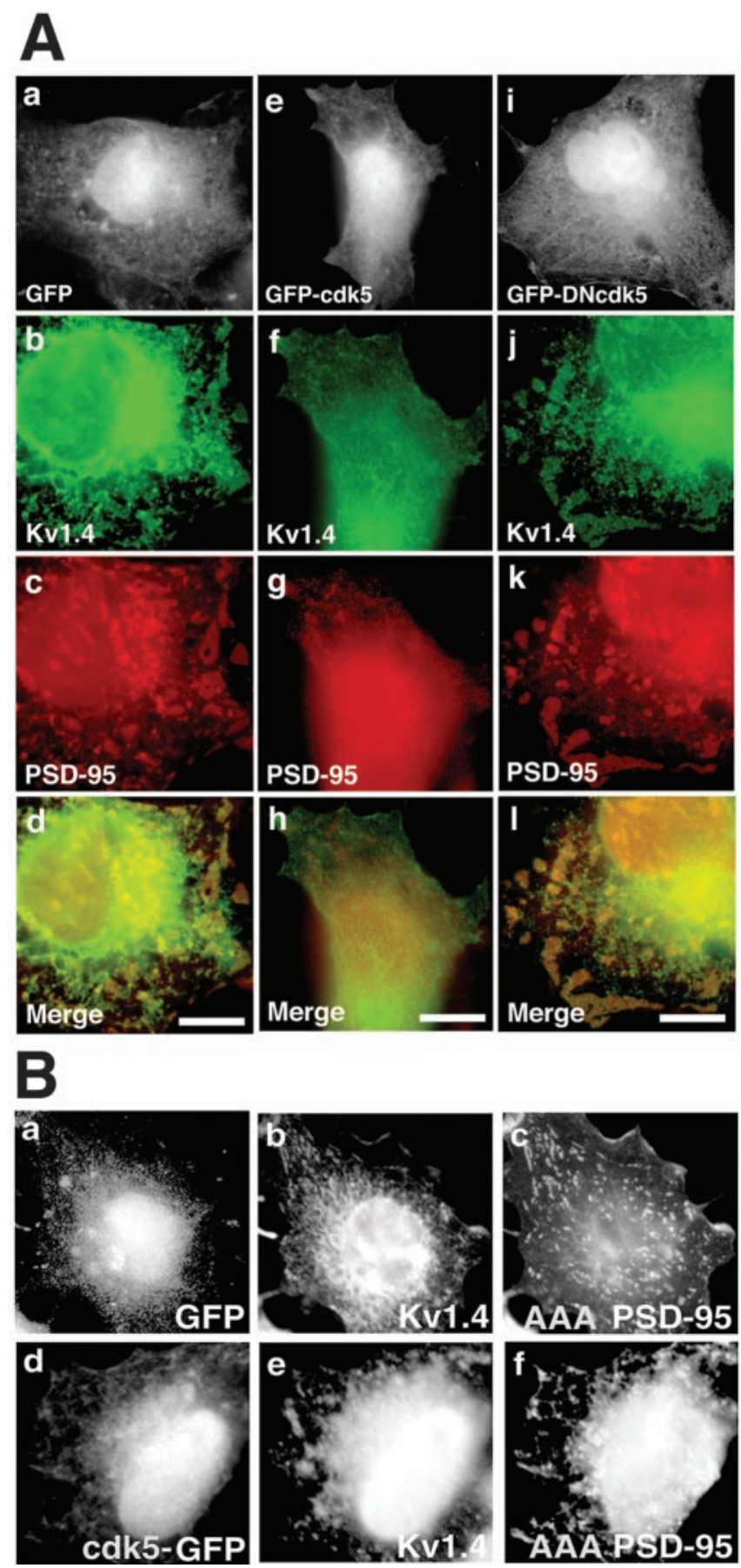

Figure 5. Active p35-cdk5 reduces the clustering of PSD-95 and ion channels in COS-7 cells. A, Clustering of PSD-95 and Kv1.4 in COS-7 cells. $a-d$, COS-7 cells cotransfected with PSD-95 (N-PDZ1-2S), $\mathrm{K}^{+}$ion channel (Kv1.4) and GFP (a) were immunostained for Kv1.4 $(b)$ and PSD-95 (c).d, Merged $b$ and c. The immunostaining is representative of $72 \%$ of transfected cells. $e-h$, COS-7 cells cotransfected with N-PDZ1-2S, Kv1.4, p35, and cdk5-GFP (e) were immunostained for Kv1.4 ( $f$ ) and PSD-95 $(g) . h$, Merged $f$ and $g$. The immunostaining is representative of $96.8 \%$ of transfected cells. $i-I$, COS-7 cells cotransfected with N-PDZ1-2S, Kv1.4, and dominant negative cdk5-GFP (DNcdk5-GFP, inactive kinase, $i$ ) were immunostained for Kv1.4 ( $j$ ) and PSD-95 ( $k$ ). $I$, Merged $j$ and $k$. The immunostaining is representative of $68 \%$ of transfected cells. Immunofluorescence images were taken by Delta Vision microscopy. Scale bars, $10 \mu \mathrm{m}$. $B$, Clustering of the T19A, S25A, S35A truncated PSD-95 mutant and Kv1.4 in COS-7 cells. $a-C$, COS-7 cells cotransfected with the T19A, S25A, T19A mutant PSD-95 (N-PDZ1-2S), Kv1.4, p35, and cdk5-GFP ( $a$ ) were immunostained for Kv1.4 (b) and PSD-95 (c). $d-f$, COS-7 cells cotransfected with the T19A, S25A, S35A mutant N-PDZ1-2S, Kv1.4, and GFP (d) were immunostained for Kv1.4 (e) and PSD-95 ( $f$. Immunofluorescence images were taken by Delta Vision microscopy. Scale bars, $10 \mu \mathrm{m}$. 

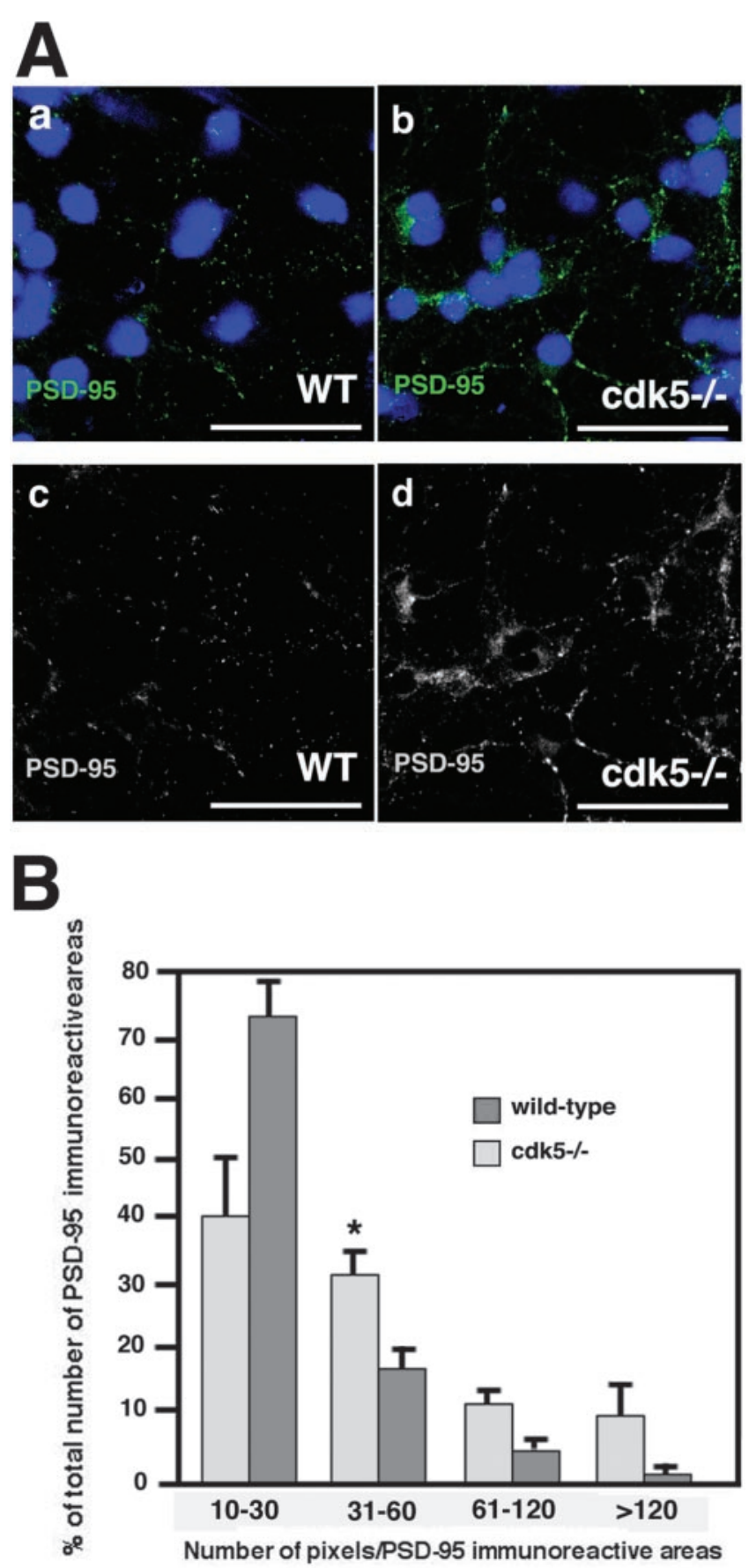

Figure 6. Cortical cdk5-/- neurons display larger PSD-95 clusters. A, PSD-95 clusters in cortical neurons (4DIV). Cortical neurons derived from wild-type (WT, $a, c)$ and cdk5 $-1-(b, d)$ E16.5 littermate embryos cultured for 4 DIV and immunostained for PSD-95 (green in $a, b ; c, d$ represent green channel only). Nuclei were stained with TOT0-3 (blue). Immunofluorescence images were taken by confocal microscopy. Scale bars, $10 \mu \mathrm{m}$. B, Morphometric analysis of areas of PSD-95 immunoreactivity in wild-type and cdk5-/- dendrites of cortical neurons and the frequency distribution of PSD-95 cluster areas as expressed in number of pixels. *Statistically significant differences between the wild-type and the $\mathrm{dk} 5-/-$ neurons.

PSD-95 clusters ranging in size from 31 to 60 pixels, 61 to 90 pixels, and clusters larger than 91 pixels constituted, respectively, $17.86 \%$ (SEM, 3.33\%), 5.40\% (SEM, 1.71\%), and 0.90\% (SEM, $0.09 \%)$ of total clusters in wild-type neurons, compared with $33.73 \%$ (SEM, 3.75\%), 11.54\% (SEM, 1.98\%), and $11.18 \%$ (SEM, 5.3\%) in cdk5-/- neurons (Fig. 6B). Together, these data argue for a regulatory role of cdk5 activity on the clustering of PSD-95 in neurons.

\section{Expression of inactive cdk5 (DNcdk5) in hippocampal neurons induces larger PSD-95 clusters}

To further determine whether cdk5 affects the synaptic clustering of PSD-95, rat hippocampal neurons were infected with recombinant HSV expressing DNcdk5-GFP (Fig. 7A, $a-c$ ), or LacZ (Fig. $7 A, d-f)$. The infection efficiencies were $30-70 \%$ as detected by either GFP or LacZ immunostaining. Clustering of PSD-95 was scored double-blind. PSD-95 immunostained clusters were detected in both DNcdk5- and LacZ (control)-expressing neurons; however, there was a notable shift toward larger clusters in DNcdk5 relative to LacZ-expressing neurons. In neurons expressing DNcdk5, an increase in the number of large PSD-95 clusters paralleled a reduction in small clusters (Fig. $7 A, a-c$ ) compared with the size distribution of clusters in LacZ-infected neurons (Fig. 7A, $d-f$ ). The size of PSD-95 clusters was quantified as described above. In the LacZ-expressing neurons, $66.18 \%$ clusters (SEM, 4.96\%) were 100-200 pixels in size, whereas only $44.03 \%$ (SEM, 3.23\%) clusters of this size were found in DNcdk5infected neurons (Fig. 7B). Conversely, in LacZ-infected neurons, $11.62 \%$ (SEM, $1.16 \%$ ) clusters were $>400$ pixels, compared with $35.86 \%$ (SEM, 0.82\%) in neurons expressing DNcdk5 (Fig. $7 B)$. Therefore, DNcdk5-GFP expression in neurons resulted in a shift from small to large clusters of PSD-95, consistent with the observation that $\mathrm{cdk} 5-/-$ cortical neurons displayed larger PSD-95 than wild-type neurons. Together, these observations are supportive of a correlation between the increase in size of PSD-95 clusters and the loss of cdk5 activity. In mature synapses, the NMDARs are clustered by PSD-95. To determine whether the increase in PSD-95 cluster size was paralleled by an increase in the size of NMDAR clusters, the hippocampal neurons expressing DNcdk5 or LacZ were immunostained with an antibody against NR1, a subunit of the NMDAR (Fig. 7C). In LacZor DNcdk5-expressing neurons, PSD-95 clusters were positive for NR1, consistent with the notion that DNcdk5 expression does not affect the association of NMDAR with PSD-95. This result also supports the hypothesis that cdk5 activity regulates clustering of PSD-95 and NMDAR at synapses.

Expression of the triple alanine mutant of PSD-95 (T19A, S25A, S35A) in hippocampal neurons induces larger PSD-95 clusters

To discriminate between a direct effect of cdk 5 phosphorylation of the N-terminal domain of PSD-95 and other effects of cdk5 and to determine whether N-terminal phosphorylation of PSD-95 is responsible for the observed reduction of clustering, rat hippocampal neurons were transfected with the triple alanine mutant (T19A, S25A, S35) of full-length PSD-95 (Fig. $8 A, a$ ), or wild-type full-length PSD-95 (Fig. 8A, $b$ ). Clustering of PSD-95 was scored double-blind. A general increase in cluster size was observed under both conditions, compared with the untransfected neurons, consistent with previous observations indicating a role of PSD-95 in the maturation of excitatory synapses (ElHusseini et al., 2000b). Interestingly, a modest but statistically significant increase in the cluster size was detected in neurons expressing the triple alanine mutant of PSD-95 compared with neurons expressing transfected wild-type PSD-95. The size of PSD-95 clusters was quantified as described above. In neurons expressing wild-type PSD-95, 51.07\% clusters (SEM, 2.27\%) were $10-30$ pixels in size, whereas only $45.25 \%$ (SEM, $1.53 \%$ ) clusters of this size were found in neurons expressing mutant 


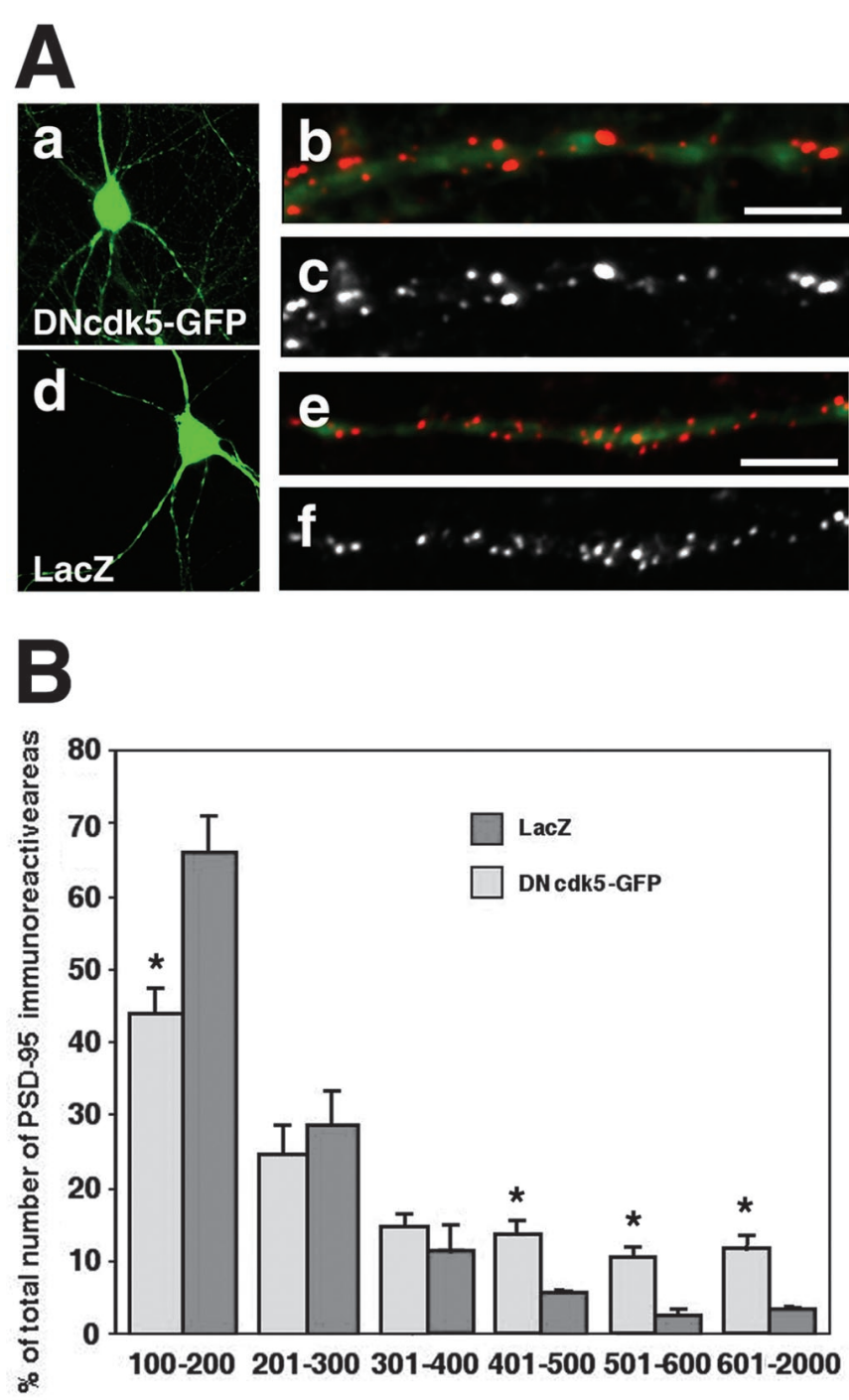

Number of pixels/PSD-95 immunoreactive areas

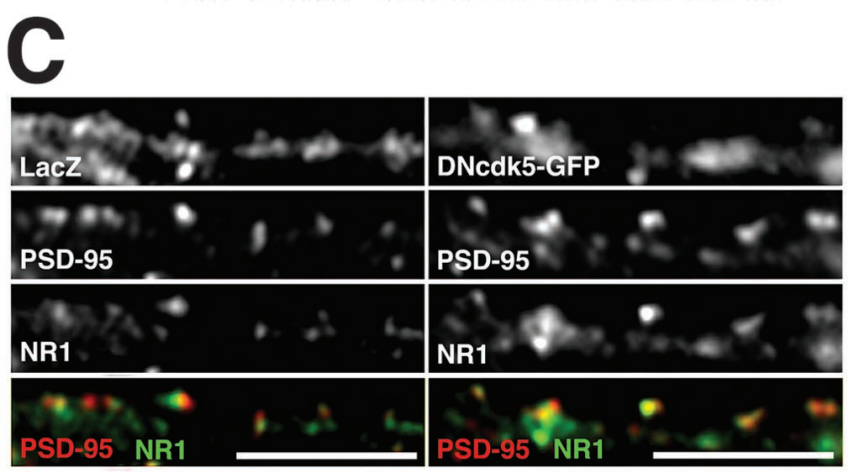

Figure 7. Expression of inactive cdk5 (DN-cdk5) in hippocampal cultures affects the clustering of PSD-95. A, $a$, GFP immunostaining of a neuron overexpressing DN-cdk5-GFP. Hippocampal neurons were cultured for 14 DIV, infected with HSV-DNcdk5-GFP, and immunostained for GFP. b, PSD-95 and GFP coimmunostaining of a neuron expressing DN-cdk5-GFP. A dendrite of HSV-DNcdk5-GFP infected hippocampal neurons immunostained for GFP (green) and PSD-95 (red). c, PSD-95 immunostaining of a neuron expressing DN-cdk5-GFP (red channel). $d, \beta$-Gal immunostaining of a neuron overexpressing LacZ. DIV 14 hippocampal neurons infected with HSV-LaCZ and immunostained for $\beta$-Gal. e, PSD-95 and $\beta$-Gal coimmunostaining of a neuron expressing LacZ. A dendrite of HSV-LacZ infected hippocampal neurons immunostained for $\beta$-Gal (green) and PSD-95 (red). f, PSD-95 immunostaining of a neuron expressing LacZ (red channel). Scale bars, $10 \mu \mathrm{m}$. B, Morphometric analysis of areas of PSD-95 immunoreactivity in neurons infected with DNcdk5-GFP or LaCZ and the frequency distribution of PSD-95 immunoreactive clusters. *Statistically significant differences between the HSV-DNcdk5-GFP versus
A
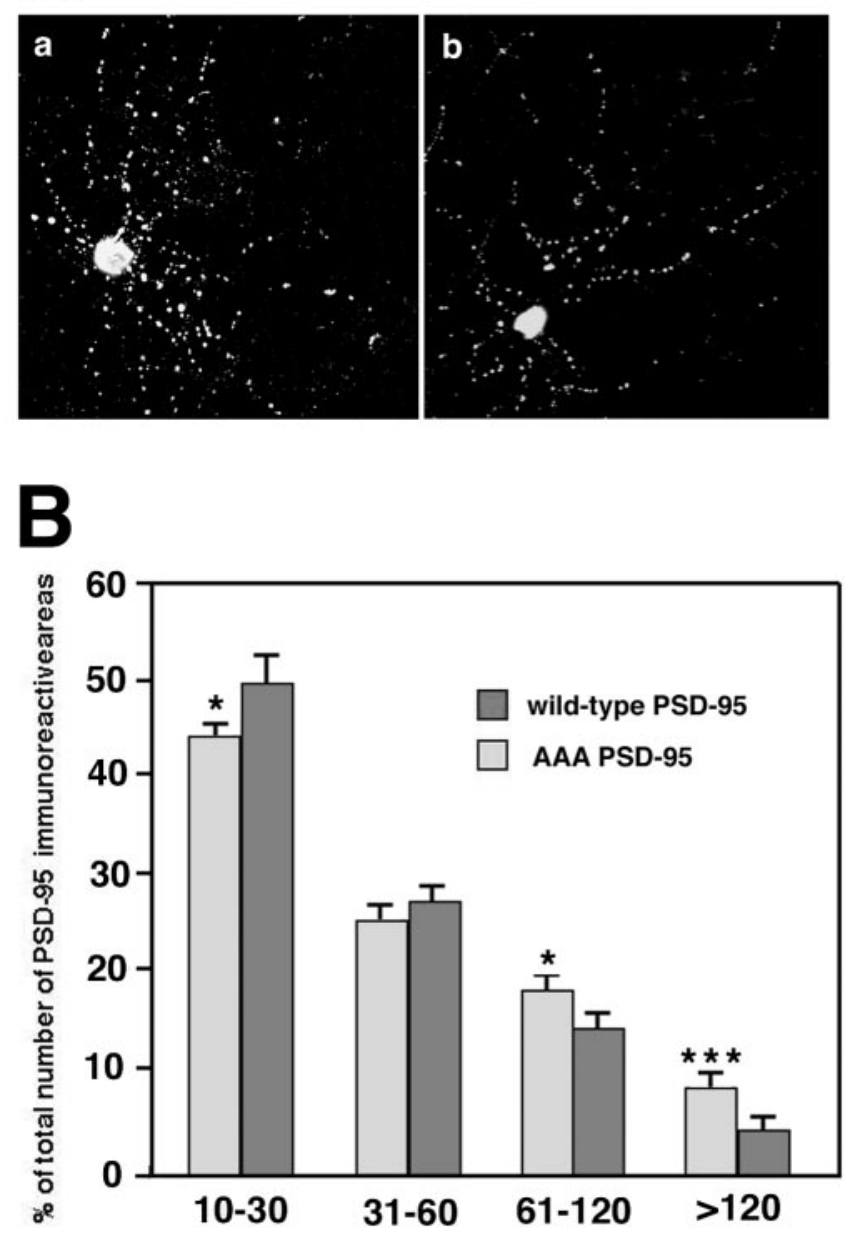

Number of pixels/PSD-95 immunoreactive areas

Figure 8. Expresssion of the triple alanine mutant (T19A, S25A, S35A) of PSD-95 in hippocampal cultures affects the clustering of PSD-95. A, $a$, PSD-95 immunostaining of a neuron expressing mutant PSD-95. Hippocampal neurons were cultured for 14 DIV, cotransfected with the triple alanine mutant (T19A, S25A, S35A) of full-length PSD-95 and GFP, and immunostained for PSD-95. b, PSD-95 immunostaining of a neuron expressing wild-type PSD-95. Scale bars, $10 \mu \mathrm{m}$. B, Morphometric analysis of areas of PSD-95 immunoreactivity in neurons transfected with mutant or wild-type PSD-95 and the frequency of distribution of PSD-95 immunoreactive cluster size. * Statistically significant differences between neurons expressing mutant versus wild-type PSD-95, as defined by the unpaired $t$ test.

PSD-95 (Fig. 8 B). Conversely, in neurons transfected with wildtype PSD-95, 15.50\% (SEM, 1.33\%) and 4.81\% (SEM, 0.69\%) of clusters were $61-120$ pixels and $>120$ pixels respectively, compared with $19.05 \%$ (SEM, 1.11\%) and 5.01\% (SEM, 0.88\%) in neurons expressing mutant PSD-95 (Fig. $8 \mathrm{~B}$ ). Consistent with the observations that cdk5-/- cortical neurons and neurons expressing DNcdk5 displayed larger PSD-95 clusters than wildtype neurons, the expression of the triple alanine mutant of PSD-95 resulted also in a shift to larger clusters of PSD-95. To-

$\leftarrow$

the HSV-LacZ infected neurons, as defined by the unpaired $t$ test. C, PSD-95 and NR1 immunostaining of infected neurons. Dendrites of hippocampal neurons infected with HSV-LacZ (left) or HSV-DNcdk5-GFP (right). Left, $\beta$-Gal immunostaining (top), PSD-95 (red) and NR1 (green) immunostaining. Right, GFP immunostaining (top), PSD-95 (red) and NR1 (green) immunostaining. Scale bars, $10 \mu \mathrm{m}$. 
gether, these observations support the model that phosphorylation of PSD-95 by cdk5 regulates the clustering of PSD-95 in neurons.

\section{Discussion}

PSD-95 has been involved in coupling the NMDARs to pathways that regulate synaptic plasticity and learning (Migaud et al., 1998). Recently, cdk5 activity has also been proposed to contribute to synaptic plasticity, learning, and memory (Li et al., 2001). We report here that PSD-95 is phosphorylated in vivo in a cdk5dependent manner, and that inhibition of cdk5 activity in neurons results in enlargement of PSD-95 clusters. These findings indicate that phosphorylation by $\mathrm{cdk} 5$ plays a regulatory role in the clustering of PSD-95 and suggest that cdk5 may contribute to the regulation of excitatory synapse formation and plasticity.

\section{PSD-95 is an in vitro substrate of cdk5}

In this study, we demonstrate that cdk5 phosphorylates PSD-95 in vitro and in vivo. Recombinant cdk5 readily phosphorylates the $\mathrm{N}$-terminal domain of PSD-95 in vitro. The specificity of the kinase reaction is upheld by the stoichiometry of 0.86 and the increase of $\gamma^{32} \mathrm{P}$ ATP incorporation observed by either increasing reaction time or amount of substrate.

\section{Phosphorylated PSD-95 is present in vivo at PSD}

To evaluate the phosphorylation of the T19 and S25 residues in vivo, we generated a phospho-epitope-specific antibody, phospho-T19S25. Western blot analysis using the phosphoT19S25 antibody revealed that phosphorylated PSD-95 is present in synaptosomes and in PSD fractions. The slower-migrating species observed in SDS-PAGE of synaptosome lysates is not detected by the phospho-T19S25 antibody and could represent the $\mathrm{N}$-terminal alternative spliced isoform of PSD-95 (Chetkovich et al., 2002). The N-terminal domain of this higher-molecularweight isoform lacks the cdk5 consensus sites, but includes an "L27" motif (Chetkovich et al., 2002), which is also present in SAP97/human discs-large (Lue et al., 1994; Muller et al., 1995), a related MAGUK protein. The hypothesis that PSD-95 is a physiological substrate of cdk5 is further supported by the drastically reduced phosphorylation of T19 and S25 in brain lysates derived from cdk5-/- mutant mice.

Phosphorylated PSD-95 is present in PSD fractions together with p35 and cdk5, supporting a role for the cdk5-dependent phosphorylation of PSD-95 in synaptic function. The $\mathrm{N}$ terminal of PSD-95 is essential for PSD-95 multimerization, membrane association, and clustering of PSD-95 and neuronal ion channels (Topinka and Bredt, 1998; Hsueh and Sheng, 1999). We find that the ability of PSD-95 to associate to membranes is decreased on phosphorylation of its $\mathrm{N}$-terminal domain, possibly because of alteration in its structure or the addition of charges to this very critical domain. The N-terminal domain of PSD-95 contains two residues, cysteines 3 and 5 that are sites of palmitoylation; this modification is thought to be essential for membrane association and ion channel binding (Topinka and Bredt, 1998). Mutations in cysteines 3 and 5 abolish the ability of PSD-95 to self-associate and to cluster ion channels (Hsueh and Sheng, 1999). It is possible that phosphorylation by cdk5 may interfere with the ability of PSD-95 to be palmitoylated or it may constitute an additional mechanism by which the $\mathrm{N}$ terminal can be post-translationally modified. Interestingly, the N-terminal domain of SAP97 does not have cysteine residues and also lacks cdk5 consensus phosphorylation sites, whereas the N-terminal domain of other synaptic MAGUKs, such as Chapsyn-110/PSD-93 (Brenman et al., 1996b; Kim et al., 1996), and SAP102 (Muller et al., 1996) include the conserved cysteine residues and potential cdk5 phosphorylation sites. It is possible that phosphorylation may be a conserved regulatory mechanism among this group of synaptic MAGUKs.

\section{Phosphorylation of PSD-95 affects its ability to multimerize but does not have an impact on binding to ion channels}

The N-terminal domain of PSD-95 is thought to be involved in PSD-95 multimerization. We show here that the ability of PSD-95 to self-associate into multimers, as detected by coimmunoprecipitation from COS-7 cell lysates, is markedly reduced on phosphorylation of the N-terminal domain of PSD-95 by cdk5. We performed these experiments using either two differentially tagged forms of PSD-95, or full-length PSD-95 together with a truncated form of PSD-95, distinguishable by electromobility. In all cases, the coexpression of PSD-95 with cdk5 reduced the ability of PSD-95 to self-associate. Consistent with this data, the multimerization of the alanine mutant of the phosphorylation sites (T19A, S25A, S35) was not affected by the expression of p35cdk5, suggesting that the phosphorylation of the N-terminal domain of PSD-95 reduces its self-association. Multimerization has been proposed as one of the mechanisms that regulates the ion channels clustering and scaffolding functions of PSD-95. We propose that cdk5-dependent phosphorylation of PSD-95 is an additional mechanism by which these functions are regulated. PSD-95 binds the NR2 subunits of the NMDAR and clusters them at synapses. Our study indicates that phosphorylation of the $\mathrm{N}$-terminal domain of PSD-95 by cdk5 does not prevent PSD-95 binding to Kv1.4 in COS-7 cells. Furthermore we observed that, in synaptosomes, phosphorylated PSD-95 associates with NR2B, a NMDAR subunit that binds PSD-95. Together, our results support the notion that multimerization and ion channel binding are two separate functions of PSD-95.

\section{Clustering of PSD-95 and ion channels is reduced by active cdk5 in heterologous cells}

The N-terminal domain of PSD-95 is essential for neuronal ion channel clustering, an important mechanism for maximizing responses to neurotransmitter release. Previous studies indicated that deletion mutants of PSD-95 lacking the N-terminal domain are unable to cluster Kv1.4 (Hsueh et al., 1997). We tested the ability of cdk5 to regulate PSD-95 and ion channel clustering in COS-7 cells. Indeed this assay revealed that clustering of both PSD-95 and Kv1.4 is remarkably disrupted in the presence of p35-cdk5, but not DNcdk5. The significance of phosphorylation of the N-terminal domain of PSD-95 on clustering is revealed by the ability of the triple alanine PSD-95 mutant (T19A, S25A, S35) to form clusters independently of p35-cdk 5 expression. The smaller size of clusters observed with these mutants is possibly caused by structural changes in the $\mathrm{N}$-terminal domain of PSD-95 that could affect its ability to bind membranes. Nonetheless, the expression of alanine PSD-95 mutant with p35-cdk5 results in visible clusters, whereas wild-type PSD-95 and p35$\mathrm{cdk} 5$ expression does not yield detectable clusters. This result is consistent with the possibility that phosphorylation of PSD-95 by p35-cdk5 regulates coclustering of PSD-95 and neuronal ion channels.

\section{Clustering of PSD-95 is increased in cdk5-/- cortical neurons}

Postsynaptic clustering is critical in both synaptogenesis and plasticity. During synapse formation, clustering of PSD-95 occurs early and may play a primary role in organizing and localiz- 
ing proteins to the PSD. PSD-95 is trafficked on dendritic endomembranes (El-Husseini et al., 2000a) and clusters at dendrites before its interaction with synaptic glutamate receptors (Rao et al., 1998). The N-terminal domain of PSD-95 has been implicated in the synaptic targeting of PSD-95. We evaluated PSD-95 clusters in young cortical cultures derived from cdk5-/- and wild-type embryonic mice littermates. The quantification of the cluster size reveals a shift toward larger clusters in cdk5-/- neurons. Because the cultures used are relatively young (only 4 DIV), the PSD-95 staining observed may reflect different stages of synaptic maturation and synaptic targeting of PSD-95. Nevertheless, these observations support a role of $\mathrm{cdk} 5$ in the regulation of clustering of PSD-95 in neurons.

\section{Expression of inactive cdk5 (DNcdk5) or triple alanine mutant PSD-95 induces larger PSD-95 clusters}

We investigated the acute effect of inhibition of cdk5 activity on mature synapses in hippocampal cultures. In these neurons, the overexpression of inactive cdk5 (DNcdk5) caused an increase in the size of PSD-95 clusters along the dendrites, indicating a deregulation of PSD-95 and NMDAR clustering in the absence of cdk5 activity. We tested the direct effect of the cdk5-dependent phosphorylation of the N-terminal domain of PSD-95 on clustering by expressing the triple alanine mutant of PSD-95 in neurons and comparing the PSD-95 clusters to those in neurons expressing wild-type PSD-95. Consistent with the analysis of cdk5-/- neurons and DNcdk5-expressing neurons, we observed an increase in cluster size along the dendrites of neurons expressing mutant PSD-95. The effect was small but statistically significant. The smaller effect observed in neurons expressing mutant PSD-95 compared with those expressing DNcdk5 could be attributed to other effects that cdk5 activity may have on the trafficking and targeting of PSD-95 and/or simply to the presence in these neurons of wild-type PSD-95, which is still able to be phosphorylated by cdk 5 and thus may reduce the effect of mutant PSD-95. An additional possibility is that the other three potential phosphorylation sites in PSD-95 (not characterized in this study) may contribute to the formation of PSD-95 clusters and their stability. Although cdk 5 activity may also have a direct impact on the formation of PSD-95 clusters, our observations suggest that cdk5-dependent phosphorylation of PSD-95 may regulate the size of the PSD dynamically. Dynamic changes in distribution and density of neuronal ion channels and receptors, and alterations in PSD structure, may play a role in synaptic plasticity (Geinisman et al., 1991; Okabe et al., 1999; Marrs et al., 2001). PSD-95 has emerged as a major player in targeting and clustering proteins at synapses and has been described as an itinerant protein (El-Husseini et al., 2000a). Interactions between the PSD-95 complex and molecular motors such as myosin-V or dynein (Naisbitt et al., 2000; Walikonis et al., 2000) may provide a driving force for PSD movements. Together, these observations support the hypothesis that clustering of PSD-95 may also be dynamic. Studies of the Drosophila neuromuscular junction indicate that calcium-calmodulin-dependent protein kinase II (CaMKII) phosphorylation regulates the distribution of the synaptic MAGUK Discs Large (DLG), which consequently has an impact on synaptic structure (Koh et al., 1999). Constitutive activation of CaMKII induces a diffuse pattern of DLG immunostaining extended to extrasynaptic regions, suggesting that anchoring of DLG at synapses is optimal when DLG is in the dephosphorylated state. Similarly, we find that inactivation of cdk5 in hippocampus neurons results in increased PSD-95 cluster size, suggesting that cdk5 may regulate clustering of PSD-95 at synapses. Inhibition of cdk5 activity by roscovitine blocks both LTP induction and NMDA-evoked currents in CA1 hippocampal neurons (Li et al., 2001). It is likely that the change in clustering of PSD-95 and NMDARs (resulting from the inhibition of cdk5 activity) may contribute to the block of LTP induction.

\section{References}

Beaudette KN, Lew J, Wang JH (1993) Substrate specificity characterization of a cdc2-like protein kinase purified from bovine brain. J Biol Chem 268:20825-20830.

Brenman JE, Chao DS, Gee SH, McGee AW, Craven SE, Santillano DR, Wu Z, Huang F, Xia H, Peters MF, Froehner SC, Bredt DS (1996a) Interaction of nitric oxide synthase with the postsynaptic density protein PSD-95 and alpha1-syntrophin mediated by PDZ domains. Cell 84:757-767.

Brenman JE, Christopherson KS, Craven SE, McGee AW, Bredt DS (1996b) Cloning and characterization of postsynaptic density 93, a nitric oxide synthase interacting protein. J Neurosci 16:7407-7415.

Brewer GJ, Torricelli JR, Evege EK, Price PJ (1993) Optimized survival of hippocampal neurons in B27-supplemented Neurobasal, a new serumfree medium combination. J Neurosci Res 35:567-576.

Chen HJ, Rojas-Soto M, Oguni A, Kennedy MB (1998) A synaptic RasGTPase activating protein (p135 SynGAP) inhibited by CaM kinase II. Neuron 20:895-904.

Chetkovich DM, Bunn RC, Kuo SH, Kawasaki Y, Kohwi M, Bredt DS (2002) Postsynaptic targeting of alternative postsynaptic density-95 isoforms by distinct mechanisms. J Neurosci 22:6415-6425.

Cho KO, Hunt CA, Kennedy MB (1992) The rat brain postsynaptic density fraction contains a homolog of the Drosophila discs-large tumor suppressor protein. Neuron 9:929-942.

Craig AM, Boudin H (2001) Molecular heterogeneity of central synapses: afferent and target regulation. Nat Neurosci 4:569-578.

Craven SE, Bredt DS (1998) PDZ proteins organize synaptic signaling pathways. Cell 93:495-498.

Craven SE, El-Husseini AE, Bredt DS (1999) Synaptic targeting of the postsynaptic density protein PSD-95 mediated by lipid and protein motifs. Neuron 22:497-509.

Dhavan R, Tsai LH (2001) A decade of cdk5. Nat Rev Mol Cell Biol 2:749-759.

El-Husseini AE, Craven SE, Chetkovich DM, Firestein BL, Schnell E, Aoki C, Bredt DS (2000a) Dual palmitoylation of PSD-95 mediates its vesiculotubular sorting, postsynaptic targeting, and ion channel clustering. J Cell Biol 148:159-172.

El-Husseini AE, Schnell E, Chetkovich DM, Nicoll RA, Bredt DS (2000b) PSD-95 involvement in maturation of excitatory synapses. Science 290:1364-1368.

Garner CC, Nash J, Huganir RL (2000) PDZ domains in synapse assembly and signalling. Trends Cell Biol 10:274-280.

Geinisman Y, DeToledo-Morrell L, Morrell F (1991) Induction of longterm potentiation is associated with an increase in the number of axospinous synapses with segmented postsynaptic densities. Brain Res 566:77-88

Hsueh YP, Kim E, Sheng M (1997) Disulfide-linked head-to-head multimerization in the mechanism of ion channel clustering by PSD-95. Neuron 18:803-814.

Hsueh YP, Sheng M (1999) Requirement of N-terminal cysteines of PSD-95 for PSD-95 multimerization and ternary complex formation, but not for binding to potassium channel Kv1.4. J Biol Chem 274:532-536.

Humbert S, Lanier LM, Tsai LH (2000) Synaptic localization of p39, a neuronal activator of cdk5. NeuroReport 11:2213-2216.

Huttner WB, Schiebler W, Greengard P, De Camilli P (1983) Synapsin I (protein I), a nerve terminal-specific phosphoprotein. III. Its association with synaptic vesicles studied in a highly purified synaptic vesicle preparation. J Cell Biol 96:1374-1388.

Irie M, Hata Y, Takeuchi M, Ichtchenko K, Toyoda A, Hirao K, Takai Y, Rosahl TW, Sudhof TC (1997) Binding of neuroligins to PSD-95. Science 277:1511-1515.

Kennedy MB (2000) Signal-processing machines at the postsynaptic density. Science 290:750-754.

Kim E, Sheng M (1996) Differential K+ channel clustering activity of PSD-95 and SAP97, two related membrane-associated putative guanylate kinases. Neuropharmacology 35:993-1000.

Kim E, Niethammer M, Rothschild A, Jan YN, Sheng M (1995) Clustering 
of Shaker-type $\mathrm{K}+$ channels by interaction with a family of membraneassociated guanylate kinases. Nature 378:85-88.

Kim E, Cho KO, Rothschild A, Sheng M (1996) Heteromultimerization and NMDA receptor-clustering activity of Chapsyn-110, a member of the PSD-95 family of proteins. Neuron 17:103-113.

Kim JH, Liao D, Lau LF, Huganir RL (1998) SynGAP: a synaptic RasGAP that associates with the PSD-95/SAP90 protein family. Neuron 20:683-691.

Ko J, Humbert S, Bronson RT, Takahashi S, Kulkarni AB, Li E, Tsai LH (2001) p35 and p39 are essential for cyclin-dependent kinase 5 function during neurodevelopment. J Neurosci 21:6758-6771.

Koh YH, Popova E, Thomas U, Griffith LC, Budnik V (1999) Regulation of DLG localization at synapses by CaMKII-dependent phosphorylation. Cell 98:353-363.

Kornau HC, Schenker LT, Kennedy MB, Seeburg PH (1995) Domain interaction between NMDA receptor subunits and the postsynaptic density protein PSD-95. Science 269:1737-1740.

Lee SH, Sheng M (2000) Development of neuron-neuron synapses. Curr Opin Neurobiol 10:125-131.

Li BS, Sun MK, Zhang L, Takahashi S, Ma W, Vinade L, Kulkarni AB, Brady RO, Pant HC (2001) Regulation of NMDA receptors by cyclindependent kinase-5. Proc Natl Acad Sci USA 98:12742-12747.

Lue RA, Marfatia SM, Branton D, Chishti AH (1994) Cloning and characterization of hdlg: the human homologue of the Drosophila discs large tumor suppressor binds to protein 4.1. Proc Natl Acad Sci USA 91:9818-9822.

Marrs GS, Green SH, Dailey ME (2001) Rapid formation and remodeling of postsynaptic densities in developing dendrites. Nat Neurosci 4:1006-1013.

McGee AW, Bredt DS (2003) Assembly and plasticity of the glutamatergic postsynaptic specialization. Curr Opin Neurobiol 13:11-18.

Migaud M, Charlesworth P, Dempster M, Webster LC, Watabe AM, Makhinson M, He Y, Ramsay MF, Morris RG, Morrison JH, O’Dell TJ, Grant SG (1998) Enhanced long-term potentiation and impaired learning in mice with mutant postsynaptic density-95 protein. Nature 396:433-439.

Milligan G, Parenti M, Magee AI (1995) The dynamic role of palmitoylation in signal transduction. Trends Biochem Sci 20:181-187.

Muller BM, Kistner U, Veh RW, Cases-Langhoff C, Becker B, Gundelfinger ED, Garner CC (1995) Molecular characterization and spatial distribution of SAP97, a novel presynaptic protein homologous to SAP90 and the Drosophila discs-large tumor suppressor protein. J Neurosci 15:2354-2366.

Muller BM, Kistner U, Kindler S, Chung WJ, Kuhlendahl S, Fenster SD, Lau LF, Veh RW, Huganir RL, Gundelfinger ED, Garner CC (1996) SAP102, a novel postsynaptic protein that interacts with NMDA receptor complexes in vivo. Neuron 17:255-265.
Mumby SM (1997) Reversible palmitoylation of signaling proteins. Curr Opin Cell Biol 9:148-154.

Naisbitt S, Valtschanoff J, Allison DW, Sala C, Kim E, Craig AM, Weinberg RJ, Sheng M (2000) Interaction of the postsynaptic density-95/guanylate kinase domain-associated protein complex with a light chain of myosin- $\mathrm{V}$ and dynein. J Neurosci 20:4524-4534.

Niethammer M, Kim E, Sheng M (1996) Interaction between the C terminus of NMDA receptor subunits and multiple members of the PSD-95 family of membrane-associated guanylate kinases. J Neurosci 16:2157-2163.

Niethammer M, Smith DS, Ayala R, Peng J, Ko J, Lee MS, Morabito M, Tsai LH (2000) NUDEL is a novel Cdk5 substrate that associates with LIS1 and cytoplasmic dynein. Neuron 28:697-711.

Nikolic M, Dudek H, Kwon YT, Ramos YF, Tsai LH (1996) The cdk5/p35 kinase is essential for neurite outgrowth during neuronal differentiation. Genes Dev 10:816-825.

Nikolic M, Chou MM, Lu W, Mayer BJ, Tsai LH (1998) The p35/Cdk5 kinase is a neuron-specific Rac effector that inhibits Pak1 activity. Nature 395:194-198.

Ohshima T, Ward JM, Huh CG, Longenecker G, Veeranna, Pant HC, Brady RO, Martin LJ, Kulkarni AB (1996) Targeted disruption of the cyclindependent kinase 5 gene results in abnormal corticogenesis, neuronal pathology and perinatal death. Proc Natl Acad Sci USA 93:11173-11178.

Okabe S, Kim HD, Miwa A, Kuriu T, Okado H (1999) Continual remodeling of postsynaptic density and its regulation by synaptic activity. Nat Neurosci 2:804-811.

Rao A, Kim E, Sheng M, Craig AM (1998) Heterogeneity in the molecular composition of excitatory postsynaptic sites during development of hippocampal neurons in culture. J Neurosci 18:1217-1229.

Sheng M, Pak DT (2000) Ligand-gated ion channel interactions with cytoskeletal and signaling proteins. Annu Rev Physiol 62:755-778.

Songyang Z, Lu KP, Kwon YT, Tsai LH, Filhol O, Cochet C, Brickey DA, Soderling TR, Bartleson C, Graves DJ, DeMaggio AJ, Hoekstra MF, Blenis J, Hunter T, Cantley LC (1996) A structural basis for substrate specificities of protein Ser/Thr kinases: primary sequence preference of casein kinases I and II, NIMA, phosphorylase kinase, calmodulin-dependent kinase II, CDK5, and Erk1. Mol Cell Biol 16:6486-6493.

Topinka JR, Bredt DS (1998) N-terminal palmitoylation of PSD-95 regulates association with cell membranes and interaction with $\mathrm{K}+$ channel Kv1.4. Neuron 20:125-134.

Walikonis RS, Jensen ON, Mann M, Provance Jr DW, Mercer JA, Kennedy MB (2000) Identification of proteins in the postsynaptic density fraction by mass spectrometry. J Neurosci 20:4069-4080. 